\title{
Spontaneous pregnancy rate after saline infusion sonogram treat done under high pressure
}

\author{
Suzan Samir Elsharkawy*
}

Department of Obstetrics and Gynecology, Faculty of Medicine, Alexandria University, Alexandria, Egypt

Received: 25 October 2019

Revised: 15 November 2019

Accepted: 19 November 2019

\section{*Correspondence:}

Dr. Suzan Samir Elsharkawy,

E-mail: samirsuzan6@gmail.com

Copyright: () the author(s), publisher and licensee Medip Academy. This is an open-access article distributed under the terms of the Creative Commons Attribution Non-Commercial License, which permits unrestricted non-commercial use, distribution, and reproduction in any medium, provided the original work is properly cited.

\begin{abstract}
Background: Failure to achieve conception after 12 months of regular unprotected intercourse is defined as infertility. The aim of this study was evaluating SIS done under high pressure (SIStreat) as a treatment procedure, for relieving simple tubal obstruction and on cumulative pregnancy rate in infertile women.

Methods: A prospective, interventional trial was done (October 2017 - November 2018) on 106 eligible women. All patients performed SIS. Patient with tubes patent under low pressure were assigned as control group, the rest of them were subjected to SIStreat, this group was farther divided into Group 2-a (patent under high pressure) and Group 2-b (occluded under high pressure). All patients had regular intercourse for 6 months. Pregnancy was confirmed by serum B-HCG.

Results: we compared patients who performed conventional SIS ( $\mathrm{n}=100$ cases) to patients who performed SIStreat afterwards $(n=84)$ according to the number of patent tubes. There was a high statistically significant difference in favor of SIStreat group $(\mathrm{p}<0.001)$. Also, there was no significant difference in pregnancy rate between control group $62.5 \%$ and Group $2-\mathrm{a} 45.7 \%(\mathrm{p}=0.226)$.

Conclusions: SIStreat is a whole new procedure for opening fallopian tubes (diagnosed occluded by SIS). Patients who were successfully treated by SIStreat had cumulative pregnancy rate comparable to patients who were diagnosed to have patent tubes using conventional SIS.
\end{abstract}

Keywords: Cumulative pregnancy rate, Infertility, Saline infusion sonogram, Transvaginal ultrasound, Tubal factor

\section{INTRODUCTION}

Failure to achieve conception after 12 months of regular unprotected intercourse is defined as infertility. ${ }^{1}$ As there are so many causes of delayed conception, mostly known and some are still unclear, tubal factor has increased since the last decade, reached up to $30-35 \%$ of cases suffering from subfertility. ${ }^{2}$ Checking tubal patency is an essential step in the investigations done for sub-fertile couples and is typically performed using hysterosalpingography (HSG) or laparoscopy with chromotubation. ${ }^{3}$ Both HSG and diagnostic laparoscopy (DL) are painful procedures, with well-known hazards of exposure to iodinated X-ray contrast media and ionizing radiation in $\mathrm{HSG}$ and possible anesthesia or operative complications in DL. ${ }^{4}$ To overcome these disadvantages, during the last two decades contrast sonography (Hy-CoSy) and saline infusion sonogram (SIS) have been used as alternatives in many countries. One advantage of these techniques is the absence of radiation; another is that these procedures can be performed in an outpatient clinic by a gynecologist. Several studies have shown that these methods have 
nearly equal sensitivity and specificity compared with laparoscopy as the golden standard for tubal patency checking. 5,6

Saline infusion sonohysterography (SIS) was first described in 1992 by Bonilla-Musoles et al, its technique entails instilling saline into the uterine cavity under ultrasound guidance; it was mainly used for assessment and better visualization of the uterine cavity. ${ }^{7-9}$ Recently, SIS was used as the main step for the assessment of fallopian tube patency, because of its simplicity, safety, and high tolerability with a very low risk of complications like; failure to complete the procedure (7\%), pelvic pain (3.8\%), vagal symptoms $(3.5 \%)$, nausea $(1 \%)$, and post procedure fever $(0.8 \%){ }^{10-13}$

The aim of this study was to evaluate SIS done under high pressure (SIStreat) as a treatment procedure, for relieving simple tubal obstruction and on cumulative pregnancy rate in women suffering from infertility. We hypothesized that SIStreat will open the tubes and increase spontaneous pregnancy rate in the treatment group.

\section{METHODS}

A prospective, interventional clinical trial was done in the period from October 2017 till November 2018 on 106 eligible women. Patients were recruited from Damanhur health insurance gynecological outpatient clinic, ElBehera governorate, Egypt. The institutional review board approved the study protocol and an informed consent was obtained from all participants prior to commencing the study.

\section{Inclusion criteria}

- Eligible patients were those women complaining of primary or secondary infertility for at least one year, with normal uterine

- Ovarian factors and semen analysis.

\section{Exclusion criteria}

- Age more than 35 or less than 18, any uterine anomalies, ovulation abnormalities,

- $\mathrm{FSH}>15 \mathrm{mIU} / \mathrm{mL}$,

- Known tubal block (diagnosed by HSG or DL) and abnormal male factor.

All patients were asked to come in the early postmenstrual period and underwent routine evaluation with complete history taking and physical examination, evaluation of the uterus and adnexa by vaginal ultrasound to exclude the presence of fluid in the cul-de-sac.

With the woman put in lithotomy position, a disposable Cusco speculum was inserted and cervix was washed with an antiseptic solution (povidone iodine). A sterile pediatric Foley catheter with a guide wire included (10 f) was inserted through cervical canal until it passed the internal os. Women with difficult procedure (due to cervical stenosis or angulation) were excluded. The catheter was flushed with sterile saline prior to insertion to clear it of air, which may cause an echogenic artifact inside the uterine cavity. Its balloon was filled with 1-2 $\mathrm{ml}$ of sterile saline solution according to uterine size and its appropriate position was confirmed by a slight pull. Careful attention was given to the exact site and amount of balloon inflation because suboptimal inflation may dislodge the catheter thus hindering proper uterine cavity distention. Also balloon over distention may hinder detection of an endometrial lesion. The speculum then was removed and a Toomey syringe filled with $60 \mathrm{ml}$ of sterile saline solution was attached to the outer end of the catheter. A transvaginal ultrasonic probe was reinserted into the posterior vaginal vault.

At first, saline was slowly infused to distend the uterus, examination of endometrial cavity for any undiagnosed lesions was done and then tubal patency was checked under low pressure that just permit proper distention and visualization. Observation of fluid in the cul de sac was considered an indication of tubal patency and the tubes were diagnosed as patent under low pressure. The procedure was repeated gently to exclude tubal spasm. If repeated injections gave similar results, the tubes were considered occluded under low pressure. Women with tubes diagnosed as occluded under low pressure were subjected to gradual increase in the infusion pressure using the Toomey syringe for 5 minutes (SIStreat), until fluid appeared in the cul de sac, otherwise the tubes were considered occluded under high pressure. Prophylactic antibiotic (100mg of doxycycline twice daily for 5 days) was prescribed and an analgesic post procedure, if needed.

Patient with tubes patent under low pressure were diagnosed as unexplained infertility (and assigned as Group 1), groups of patients with tubes which are occluded under low pressure are assigned as Group 2 (SIStreat group), this group was farther divided into Group 2-a (patients with tubes patent under high pressure) and Group 2-b (patients who were diagnosed with tubes occluded under high pressure). All patients were advised to have regular intercourse in fertile periods for the next 6 months of follow up. Pregnancy was confirmed by serum B-HCG after a missed period and with ultrasonic diagnosis of intact intrauterine gestational sac one week afterwards.

The primary outcome was opening the fallopian tubes evidenced by passage of saline into pouch of Douglas detected by TV-US. Secondary outcome was the occurrence of spontaneous clinical pregnancy detected by a sonographically visible gestational sac within the follow up period of six months. The results of all groups were compared to each other. 


\section{Statistical analysis}

Data were fed to the computer and analyzed using IBM SPSS software package version 20.0. (Armonk, NY: IBM Corp). Qualitative data were described using number and percent. Significance of the obtained results was judged at the $5 \%$ level. ${ }^{14,15}$

\section{RESULTS}

The total number of women included in the study was 106 patients who suffered from primary or secondary infertility for at least one year, with normal uterine, ovarian and male factors. 6 cases were missed during the follow up period and 100 cases completed the study. Age, parity and number of children did not differ significantly among groups, as shown in Table 1.

All cases performed SIS in the early postmenstrual period as a diagnostic method for evaluation of tubal patency. Out of the 100 cases that completed the study, 16 cases had tubes diagnosed to be patent under low infusion pressure (evidenced by the visualization of saline in the cul de sac by TV-US), these patients were considered suffering from unexplained infertility, assigned as Group1 and were subjected to expectant management for 6 months.

Table 1: Comparison between the three studied groups according to age, parity and number of children.

\begin{tabular}{|c|c|c|c|c|c|}
\hline & Group $1(n=16)$ & Group 2-a $(n=70)$ & Group 2-b $(n=14)$ & Test of Sig. & p \\
\hline \multicolumn{6}{|l|}{ Age (years) } \\
\hline Min.-Max. & $19-34$ & $19-39$ & $20-35$ & \multirow{3}{*}{$\mathrm{F}=1.228$} & \multirow{3}{*}{0.297} \\
\hline Mean \pm SD & $26.3 \pm 5.3$ & $28.2 \pm 6$ & $29.4 \pm 4.7$ & & \\
\hline Median (IQR) & $27(19.8-30.3)$ & $27(21-32.8)$ & $30(28-34)$ & & \\
\hline \multicolumn{6}{|l|}{ Parity } \\
\hline Nulliparas & $6(37.5 \%)$ & $20(28.6 \%)$ & $6(42.9 \%)$ & \multirow{2}{*}{$\chi^{2}=1.359$} & \multirow{2}{*}{0.507} \\
\hline Gravidas & $10(62.5 \%)$ & $50(71.4 \%)$ & $8(57.1 \%)$ & & \\
\hline \multicolumn{6}{|c|}{ Number of children } \\
\hline Min.-Max. & $0-3$ & $0-5$ & $0-2$ & \multirow{3}{*}{$\mathrm{H}=1.701$} & \multirow{3}{*}{0.427} \\
\hline Mean \pm SD & $1 \pm 1$ & $1.1 \pm 1$ & $0.7 \pm 0.7$ & & \\
\hline Median (IQR) & $1(0-1.5)$ & $1(0.3-2)$ & $1(0-1)$ & & \\
\hline
\end{tabular}

$\chi^{2}$ : Chi square test, F: $\mathrm{F}$ for ANOVA test, $\mathrm{H}: \mathrm{H}$ for Kruskal Wallis test, $\mathrm{p}$ : P-value for comparing between the studied groups, *: Statistically significant at $\mathrm{p} \leq 0.05$

Table 2: Comparison between SIS and SIStreat according to patency of tubes.

\begin{tabular}{|lllll|}
\hline & SIS $(\mathbf{n}=\mathbf{1 0 0})$ & SIStreat $(\mathbf{n}=\mathbf{8 4})$ & $\chi^{\mathbf{2}}$ & $\mathbf{p}$ \\
\hline Patent & 16 & 70 & $83.144^{*}$ & $<0.001 *$ \\
\hline Occluded & 84 & 14 & \\
\hline
\end{tabular}

$\chi^{2}$ : Chi square test, $*$ : Statistically significant at $\mathrm{p} \leq 0.05$

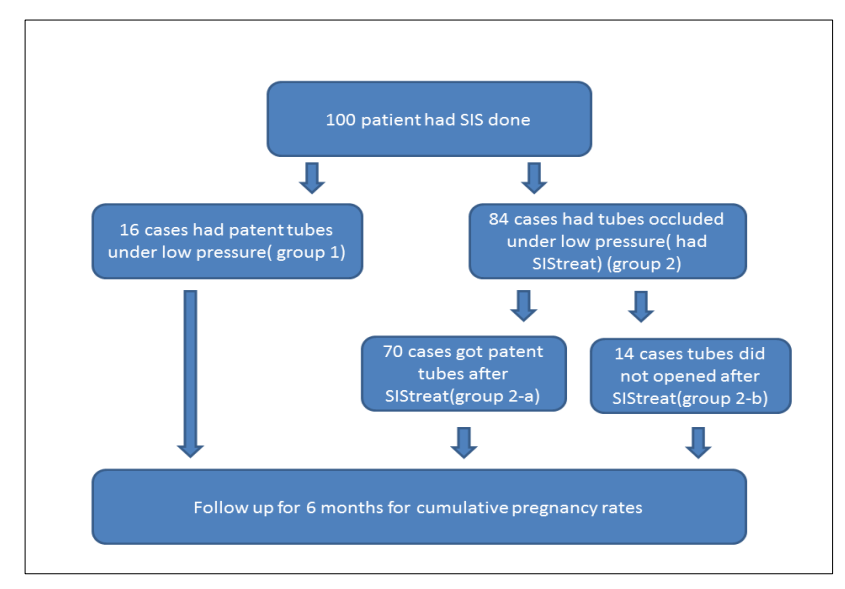

Figure 1: Schematic representation of the different study groups.
Meanwhile the remaining cases (Group 2; 84 cases), had tubes that did not pass saline under low infusion pressure, and where subjected to gradual increase in infusion pressure for 5 minutes in the same sitting as a trial for treatment of the tubal occlusion (SIStreat). In 70 cases, the tubes opened under high infusion pressure evidenced by the visualization of saline in the cul de sac by TV-US, these patients were assigned as group 2-a. While in 14 cases, the tubes remained closed after SIStreat and assigned as Group 2-b. all patients were followed up for 6 months afterword's for spontaneous pregnancy. as shown in Figure 1.

To evaluate SIStreat efficacy to open the tubes that were diagnosed occluded after regular SIS; we compared patients who performed conventional SIS ( $n=100$ cases) to patients who performed SIStreat afterwards $(n=84)$ 
according to the number of patent tubes after application of the two procedures. There was a high statistically significant difference in favor for SIStreat group ( $\mathrm{p}<$ 0.001 ), as shown in Table 2.

After 6 months of follow up of the three groups (1, 2-a, 2b) the cumulative pregnancy rate for Group 1 was $62.5 \%$ (10 cases out of 16), and for Group 2-a who was subjected to successful SIStreat was $45.7 \%$ (32 cases out of 70), while for Group 2-b was $14.2 \%$ (2 cases out of 14). When statistically compared to each other; there was no significant difference in pregnancy rate between Group 1 and Group 2-a $(\mathrm{p}=0.226)$, but when both groups (1 and 2-a) were compared to Group 2-b, there were statistically significant differences; $(\mathrm{p}=0.007)$ and $(\mathrm{p}=0.029)$ respectively, as shown in Table 3 .

Table 3: Comparison between the three studied groups according to cumulative pregnancy rate.

\begin{tabular}{|c|c|c|c|c|c|}
\hline & Group $1(n=16)$ & Group 2-a $(n=70)$ & Group 2-b $(n=14)$ & Test of Sig. & p \\
\hline \multicolumn{6}{|l|}{ Pregnancy } \\
\hline Non pregnant & $6(37.5 \%)$ & $38(54.3 \%)$ & $12(85.7 \%)$ & \multirow{2}{*}{$\begin{array}{l}\chi^{2}= \\
7.323^{*}\end{array}$} & \multirow{2}{*}{$0.026^{*}$} \\
\hline Pregnant & $10(62.5 \%)$ & $32(45.7 \%)$ & $2(14.3 \%)$ & & \\
\hline Significance between groups & \multicolumn{5}{|c|}{$\mathrm{p}_{1}=0.226, \mathrm{p}_{2}=0.007^{*}, \mathrm{p}_{3}=0.029^{*}$} \\
\hline \multicolumn{6}{|c|}{$\begin{array}{l}\chi^{2} \text { : Chi square test, } \mathrm{H}: \mathrm{H} \text { for Kruskal Wallis test, Pairwise comparison between each } 2 \text { groups was done using Post Hoc Test (Dunn's } \\
\text { for multiple comparisons test), } \mathrm{p} \text { : } \mathrm{p} \text { value for comparing between the studied groups, } \mathrm{p} 1 \mathrm{p} \text { value for comparing between gr } 1 \text { and gr } \\
\text { 2-a, p2: } \mathrm{p} \text { value for comparing between gr } 1 \text { and gr } 2-\mathrm{b}, \mathrm{p} 3 \text { : } \mathrm{p} \text { value for comparing between gr } 2-\mathrm{a} \text { and gr } 2 \text {-b, *: Statistically } \\
\text { significant at } \mathrm{p} \leq 0.05 \text {. }\end{array}$} \\
\hline
\end{tabular}

\section{DISCUSSION}

Checking tubal patency is an essential step in management of infertile couples, as tubal factor is responsible for around $25-35 \%$ of cases of delayed conception and is considered as a major indication for ICSI treatments. ${ }^{16,17}$

To test the tube patency, you have to push something to pass through. That is why all available methods are considered invasive, ranging from SIS, HSG up to laparoscopy. Spontaneous pregnancy rates increase after HSG, which may be due to the effect of tubal flushing that will break minor adhesions or sweep mucus plugs and cell debris through the tubes. A very recent metaanalysis has declared that the use of an oil-soluble contrast medium showed a significantly higher clinical pregnancy rate within 6 months, than a water-soluble contrast medium (WSCM) or no flushing at all. ${ }^{18}$ On the other hand, SIS was found to increase pregnancy rates when it was performed prior to ICSI cycles if there were no associated uterine pathology. ${ }^{19}$

Our idea originated from using SIS as a simple therapeutic method for tubal factor infertility, by increasing infusion pressure gradually to wash out any debris, mucous or minor tubal adhesions that may hinder pregnancy, and we called it "SIStreat". First, we compared the patients who were diagnosed to have patent tubes after using regular SIS (control group) with the patients had their tubes opened after using SIStreat (treatment group) to evaluate the procedure efficacy. Then we compared the cumulative pregnancy rates after 6 months of follow-up for all groups. Our results showed that SIStreat succeeded to open $83 \%$ of the tubes diagnosed "occluded" by regular SIS, with statistically significant difference between the two methods in favor of SIStreat. Pregnancy rate in the treatment group tubes did not differ from the control group, reaching $45.7 \%$ (32 cases out of 70 ) and $62.5 \%$ (10 cases out of 16) respectively, which proves that the new method was successful in treating these women. The pregnancy rate in the group of patients that SIStreat failed to open their tube was $14.2 \%$ ( 2 cases out of 14$)$, which had statistically significant difference from the "SIStreated" group $(\mathrm{p} 3=0.029)$. This result may be due to transient block due to tubal spasm that led to failure of SIStreat to open up the tubes during the procedure.

According to our knowledge, this is the first time to use saline infusion sonogram as a treatment procedure. As HSG did before, SIStreat can break fine adhesions, removes mucous plugs and cleans cell debris. Another mechanism may explain our results; SIStreat may cause endometrial damage due to catheterization process and cavity distension, which caused cytokines and growth factors secretion (e.g., leukemia inhibitory factor, interleukin-11, and heparin-binding endothelial growth factor) that are proved to be essential for embryo implantation. ${ }^{20-22}$

The drawback of this study was the fact that the treatment group had performed SIStreat after they did SIS in the same setting. Although this may influence our results a little, but we considered this to be an advantage for the procedure, which can be used for diagnosis and therapy in one clinic visit, without wasting time and money in multiple visits. ${ }^{23-25}$

According to our experience, the technique was easy and well accepted by the patients, and only mild pelvic discomfort was noted. Originally, SIS is an outpatient procedure that is easily executed, cheap, safe, less invasive, does not require sedation or anesthesia, has no 
risk of exposure to ionizing radiation, better tolerated diagnostic tool and has a short learning curve from 2-9 times for trainers. If it is possible to use this perfect procedure as an instant treatment for mild tubal factor, why not?.

\section{CONCLUSION}

SIStreat is a whole new, effective, easy and cheap procedure for opening fallopian tubes that were diagnosed occluded by SIS. Patients who were successfully treated by SIStreat had cumulative pregnancy rate comparable to patients who were diagnosed to have patent tubes using conventional SIS.

Funding: No funding sources

Conflict of interest: None declared

Ethical approval: The study was approved by the Institutional Ethics Committee

\section{REFERENCES}

1. ESHRE Capri Workshop Group. Diagnosis and management of the infertile couple: missing information. Hum Reprod Update. 2004;10:295-307.

2. Kupesic S, Kurjak A. Interventional ultrasound in human reproduction. In: Kupesic S, De Ziegler D, editors. Ultrasound and Infertility. New York, NY: Parthenon Publishing; 2000:253-263.

3. National Institute for Health and Clinical Excellence: Fertility: assessment and treatment for people with fertility problems. Nice clinical guideline 156; 2013. Available at: guidance.nice.org.uk/cg156.

4. Exacoustos C, Zupi E, Carusotti C, Lanzi G, Marconi D, Arduini D. Hystero-salpingo-contrastsonography compared with hysterosalpingography and laparoscopic dye perturbation to evaluate tubal patency. J Am Assoc Gynecol Laparosc. 2003;10:2932.

5. Dietrich M, Suren A, Hinney B, Osmers R, Kuhn W. Evaluation of tubal patency by hysterocontrast sonography (HyCoSy, Echovist) and its correlation with laparoscopic findings. J Clin Ultrasound. 1996;24:523-7.

6. Testa AC, Ferrandina G, Fruscella E, Van Holsbeke C, Ferrazzi E, Leone FP, et al. The use of contrasted transvaginal sonography in the diagnosis of gynecologic diseases: a preliminary study. J Ultrasound Med. 2005;24:1267-78.

7. Bonilla-Musoles F, Simon C, Serra V, Sampaio M, Pellicer A. An assessment of hysterosalpingosonography (HSSG) as a diagnostic tool for uterine cavity defects and tubal patency. J Clin Ultrasound. 1992;20:175-81.

8. Parsons AK, lense JJ. Sonohysterography for endometrial abnormalities: preliminary results. J Clin Ultrasound. 1993;21:87-95.

9. Goldstein SR. Saline infusion sonohysterography. Clin Obstet Gynecol. 1996;39:248-58.
10. Hajishafiha M, Zobairi T, Zanjani VR, Ghasemi-Rad M, Yekta Z, Mladkova N. Diagnostic value of sonohysterography in the determination of fallopian tube patency as an initial step of routine infertility assessment. J Ultrasound Med. 2009;28(12):1671-7.

11. Malek-Mellouli M, Gharbi H, Reziga H. The value of sonohysterography in the diagnosis of tubal patency among infertile patients. Tunis Med. 2013;91(6):387-90.

12. Saunders RD, Shwayder JM, Nakajima ST. Current methods of tubal patency assessment. Fertil Steril. 2011;95:2171-9.

13. Dessole S, Farina M, Rubattu G, Cosmi E, Ambrosini G, Nardelli GB. Side effects and complications of sonohysterosalpingography. Fertil Steril. 2003;80(3):620-4.

14. Kotz S, Balakrishnan N, Read CB, Vidakovic B. Encyclopedia of statistical sciences. $2^{\text {nd }}$ ed. Hoboken, N.J.: Wiley-Interscience; 2006.

15. Kirkpatrick LA, Feeney BC. A simple guide to IBM SPSS statistics for version 20.0. Student ed. Belmont, Calif.: Wadsworth, Cengage Learning; 2013.

16. Strandell A, Waldenström U, Nilsson L, Hamberger L. Hydrosalpinx reduces in-vitro fertilization/embryo transfer pregnancy rates. Human Repro. 1994;9(5):861-3.

17. Berek JS. Berek and Novak's Gynecology. $14^{\text {th }}$ ed. Philadelphia, PA: Lippincott Williams and Wilkins; 2007.

18. Wang R, van Welie N, van Rijswijk J, Johnson NP, Norman RJ, Dreyer K, et al. Effectiveness on fertility outcome of tubal flushing with different contrast media: systematic review and network meta-analysis. Ultrasound Obstet Gynecol 2019;54:172-81.

19. Sefa K, Ciragil G, Yücel B, Gencdal S, Destegul E, Ozdemir O. Saline infusion sonography can improve outcome in subsequent ICSI cycles when no intrauterine pathology is detected. J Reprod Med. 2016;61(5):421-4.

20. Zhou L, Li R, Wang R, Huang HX, Zhong K. Local injury to the endometrium in controlled ovarian hyperstimulation cycles improves implantation rates. Fertil Steril. 2008;89:1166-76.

21. Sherer DM, Abulafia O. Angiogenesis during implantation, and placental and early embryonic development. Placenta. 2001;22:1-3.

22. Mirkin S, Nikas G, Hsiu JG, Díaz J, Oehninger S. Gene expression profiles and struc-tural/functional features of the peri-implantation endometrium in natural and gonadotropin-stimulated cycles. J Clin Endocrinol Metab. 2004;89:5742-52.

23. Soares SR, Barbosa dos Reis MMB, Camargos AF. Diagnostic accuracy of sonohysterography, transvaginal sonography, and hysterosalpingography in patients with uterine cavity diseases. Fertil Steril. 2000;73:406-11.

24. Inki P, Palo P, Antlia L. Vaginal sonosalpingography in the evaluation of tubal patency. Acta Obstet Gynecol Scand. 1998;77:975-82. 
25. Romano F, Cicinelli E, Anastasio PS, Epifani S, Fanelli F, Galantino P. Sonohysterography versus hysteroscopy for diagnosing endouterine abnormalities in fertile women. Int J Gynecol Obstet. 1994;45:253-60.
Cite this article as: Elsharkawy SS. Spontaneous pregnancy rate after saline infusion sonogram treat done under high pressure. Int J Reprod Contracept Obstet Gynecol 2020;9:255-60. 